\title{
Genetic alterations in primary osteosarcoma from 54 children and adolescents by targeted allelotyping
}

\author{
N Entz-Werle ${ }^{*, 1-10}$, A Schneider', C Kalifa ${ }^{2}$, A-C Voegeli', M-D Tabone ${ }^{3}$, P Marec-Berard ${ }^{4}$, L Marcellin ${ }^{5}$, \\ H Pacquement ${ }^{6}$, P Terrier ${ }^{7}$, P Boutard ${ }^{8}$, N Meyer ${ }^{9}$, M-P Gaub ${ }^{1}$, P Lutz $^{10}$, A Babin ${ }^{10}$ and P Oudet \\ 'Service de Biochimie et Biologie Moléculaire, CHRU Hautepierre, Avenue Molière, 67098 Strasbourg Cedex, France; '²ervice de Pédiatrie Onco- \\ Hématologie, Institut Gustave Roussy, 39 rue Camille Desmoulins, 94809 Villejuif Cedex, France; ${ }^{3}$ Service de Pédiatrie Onco-Hématologie, Hôpital \\ Trousseau, 26 avenue du Dr Netter, 7557 I Paris Cedex 12, France; ${ }^{4}$ Service d'Oncologie Pédiatrique, Centre anticancéreux Léon Berard, 28 rue \\ Laennec, Lyon, France; ${ }^{5}$ Senvice d'Anatomopathologie, CHRU Hautepierre, Avenue Molière, 67098 Strasbourg Cedex, France; ' $5 e n v i c e$ d'Oncologie \\ Pédiatrique, Institut Curie, - 26 rue d'Ulm, 7523I Paris Cedex, France; 'Senvice d'Anatomopathologie, Institut Gustave Roussy, 39 rue Camille Desmoulins, \\ 94809 Villejuif Cedex, France; ${ }^{8}$ Senvice d'Onco-Hématologie Pédiatrique, CHRU Caen, Avenue de la Côte de Nacre, 14033 Caen Cedex, France; \\ ${ }^{9}$ Laboratoire de Biostatistique et informatique Médicale, Département d'Information Médicale, CHRU Strasbourg I place de l'Hôpital, 6709 I Strasbourg, \\ France; ${ }^{10}$ Service de Pédiatrie Onco-Hématologie, CHRU Hautepierre, Avenue Molière, 67098 Strasbourg Cedex, France
}

\begin{abstract}
At present, the only recognised prognostic factor for primary osteosarcoma is the histological response to preoperative chemotherapy. Our study was designed to identify new diagnostic markers that could eventually have a prognostic value. A total of 54 patients under 20 years of age with primary osteosarcomas were studied while under treatment by the French Society of Paediatric Oncology OS 94 protocol. Paired normal and biopsy samples were collected. In addition, surgical resection specimens, following preoperative chemotherapy, were obtained in 13 cases. After genomic DNA extraction, an allelotyping analysis targeting microsatellites linked to Rb and p53 genes, and 9p2 I, 7q31 and 5q2I regions was performed. In all, 94\% of the samples at diagnosis showed allelic imbalance and the biopsies were highly rearranged except for the microsatellite targeting 7q31. The same panel was highly informative at surgical resection. Microsatellites investigating Rb, p53 and the 9p2I region were particularly altered without a significant correlation with prognosis. On the other hand, the alteration of the $7 q 31$ locus at diagnosis was significantly correlated with a worse prognosis and a new frequently altered locus, 5q21, was described. In conclusion, this panel allowed us to characterise paediatric osteosarcomas. Correlation of prognosis with the altered $7 q 31$ region could be a useful tool and further studies are required to confirm the importance of $5 \mathrm{q} 21$.

British Journal of Cancer (2003) 88, 1925-1931. doi:10.1038/sj.bjc.6600968 www.bjcancer.com
\end{abstract}

(c) 2003 Cancer Research UK

Keywords: high-grade osteosarcoma; microsatellites; paediatric population; Rb; p53; 5q2I region; 7q3I region

Primary high-grade osteosarcoma is the most frequent malignant bone cancer in childhood accounting for about $5 \%$ of all paediatric solid tumours. It is diagnosed mainly in teenagers and mostly localised in the long bones of limbs. Anatomopathological analysis of tumour biopsy confirms only the diagnosis of high-grade osteosarcoma without any prognostic consequence (Dahlin and Unni, 1986; Huvos, 1991). In one study, the site of the primary tumour had no correlation with survival (Nagler et al, 2000), whereas others have reported a significant trend towards survival in the axial or appendicular flat bone high-grade osteosarcomas (Duffaud et al, 2000; Gadwal et al, 2001).

In this study, after diagnosis, the patients were treated with the OS 94 protocol, which included preoperative chemotherapy, followed by surgery and postoperative chemotherapy. In this protocol of the French Society of Paediatric Oncology (SFOP), a randomised preoperative chemotherapy was administered. The

*Correspondence: Dr N Entz-Werle, Service de Biochimie et Biologie Moléculaire, Service d'Onco-Hématologie Pédiatrique, CHRU Hautepierre, Avenue Molière, 67098 Strasbourg Cedex, France;

E-mail: Natacha.Entz-werle@chru-strasbourg.fr

Received 2 September 2002; revised 3 February 2003; accepted 6 March 2003 first line combined either anthracycline and high-dose methotrexate or etoposide, ifosfamide and high-dose methotrexate. After surgery, patients were classified as good (GR) or poor responders (PR) to preoperative chemotherapy according to the histological grading system established by Huvos et al (1977). Postoperatively, GR were treated with the same drugs and PR received a second-line chemotherapy. Assessment of histological response to preoperative chemotherapy is currently the only major prognostic tool. Thus, a clear need exists for early identification of patients that would relapse or fail to respond. Until now, the validation of prognostic diagnostic factors has been unsuccessful.

In the majority of primary osteosarcomas, the aetiology is unknown, but, for a minority, it occurs in the setting of a previous cancer, such as a hereditary mutation of retinoblastoma or an autosomic recessive mutation of p53 in the Li-Fraumeni syndrome (Huvos et al, 1977; Malkin et al, 1990; Alonso et al, 2001). These two genes, localised in 13q14 and 17p13, respectively, could therefore be involved in the multistep oncogenesis model of high-grade osteosarcomas. Indeed, the currently accepted model of oncogenesis corresponds to a stepwise accumulation of genomic defects.

Until now, the histological analysis at diagnosis and on surgical resection has been insufficient in explaining the oncogenesis of osteosarcomas and the worse evolution of some GR. Immunohistochemistry has allowed analysis of the expression of 
several protein targets, like proteins involved in cell cycle or differentiation, but with a low sensitivity (Wadayama et al, 1994; Ferracini et al, 1995; Nielsen et al, 1998; Maitra et al, 2001; Park et al, 2001). Likewise, cytogenetic studies of osteosarcomas have shown various complex cytogenetic changes involving some chromosomes but without any specific pattern (Mertens et al, 1993; Ozisil et al, 1994). At the molecular level, some of the biological factors proposed thus far as potential prognostic factors in osteosarcoma are p15, p16 (Nielsen et al, 1998; Maitra et al, 2001), p53 (Park et al, 2001), Rb (Wadayama et al, 1994) and c-met (Ferracini et al, 1995; Oda et al, 2000), but these studies have mainly been performed on small populations.

Allelotyping analyses by identification of microsatellite modifications could reveal directly at the DNA level the presence of chromosomal alterations and could characterise either chromosomal (allelic imbalance or AI) or microsatellite instabilities (MSIs). The presence of MSIs identifies the repair error phenotype (RER) (Karran, 1996). Although the allelotyping is a powerful and sensitive method, it does not establish whether allelic imbalances correspond to allelic gains or losses. Microsatellite studies can however allow the characterisation of some loci potentially involved in the multistep carcinogenesis, like p16, p53 and Rb genes. These were the cell cycle genes shown to be most frequently altered in osteosarcomas in previous studies, but, with controversial prognostic implications (Tsuchiya et al, 2000; Maitra et al, 2001; Park et al, 2001). A French working group has produced a first retrospective molecular study, showing a significant trend in the association of alteration at $\mathrm{Rb}$ locus and a bad prognosis (Feugeas et al, 1996). Up to now, the status of many differentiation and proliferation genes such as c-met or APC, which are known to be involved in other cancers have not yet been analysed in paediatric osteosarcomas. Our study, including 54 patients, was therefore designed to identify prognostic factors assessable at diagnosis in osteosarcoma by analysing the microsatellite status of $5 q 21,7 q 31,9 p 21,13 q 14$ and 17 p13 loci. Furthermore, the results were correlated with the histological response to preoperative chemotherapy and survival.

\section{PATIENTS AND METHODS}

\section{Patients}

In total, 54 patients (Table 1) with primary high-grade osteosarcoma were included in our molecular study. All were treated following the guidelines of the OS 94 protocol between November 1994 and October 2001. Six patients had a metastatic disease at diagnosis (patients 16, 19, 37, 42, 49 and 53). The population consisted of 30 GR and $23 \mathrm{PR}$, according to the histopathological grading reported by Huvos et al (1977). Only one patient was not assessable. The GR patients were defined with a necrosis cutoff of $95 \%$ or more. This population comprised 30 boys and 24 girls. The median age at diagnosis was 13 years old (range from 4 to 20 years). The initial tumour localisation consisted of 31 femurs, 12 tibias, two fibulas, five humerus, one mandibular and two pelvises. In one case, these data were not assessable. At the end of our study, among these 54 patients, 42 are in first continuous complete remission (CCR1), two in second continuous complete remission (CCR2), two in partial remission, one in relapse and seven died. All deaths were because of progressive and metastatic osteosarcomas, highly resistant to chemotherapy. No toxic death was observed in this cohort. The median survival was 57 months (range from 3 to 86 months).

\section{Sample collection for molecular analysis}

Biopsies from 54 patients were collected at diagnosis, fresh-frozen and stored at $-80^{\circ} \mathrm{C}$. The biopsies were histologically characterised by a pathologist. In addition, samples from the surgical resection, following the preoperative chemotherapy, were collected in 13 cases. The histological grading was made in these 13 cases according to the previously defined necrosis cutoff. For one patient, we obtained the relapse tissue and for another, the metastatic specimen. Paired normal tissue was obtained from peripheral blood samples conserved on Whatman paper.

\section{METHODS}

DNA extraction Tissue and blood paired DNA were purified as described in Miller et al (1988) and Schneider et al (2000). Tumour and blood genomic DNA concentrations ranged from 50 to $400 \mathrm{ng} \mu \mathrm{l}^{-1}$ and from 1 to $10 \mathrm{ng} \mu \mathrm{l}^{-1}$, respectively.

Microsatellite analysis Six microsatellites were analysed on paired normal and biopsy DNA. They were informative for p53 (TP53), localised in the $\mathrm{Rb}$ gene ( $R B 1$, intron 20$)$, in the $9 \mathrm{p} 21$ (D9S171) and 7q31 (D7S486) regions and at the 5q21 locus. D5S346 and D5S492 at 5q21 were centromeric and telomeric, respectively, in a region containing the APC gene among others. Sequences of primers were obtained from the genomic database (http:// www.ncbi.nlm.nih.gov/genemap99 and http://www.gdb.org). Genomic DNA from both the paired samples (10 $\mathrm{ng}$ ) was amplified by PCR in a total volume of $50 \mu \mathrm{l}$ using $1.2 \mathrm{U}$ of Taq polymerase and 4 pmol of both forward and Cy5 labelled reverse primers. PCR was carried out in an Omnigen Hybaid Thermocycler (Hybaid Ltd, Ashford, UK) using the following protocol: $7 \mathrm{~min}$ at $95^{\circ} \mathrm{C}, 35$ cycles of $1 \mathrm{~min}$ at $95^{\circ} \mathrm{C}, 1 \mathrm{~min}$ at $55^{\circ} \mathrm{C}(D 9 S 171, D 5 S 346, D 5 S 492, T P 53)$ and $53^{\circ} \mathrm{C}(R B 1, D 7 S 486)$ and $1 \mathrm{~min}$ at $72^{\circ} \mathrm{C}$, followed by $5 \mathrm{~min}$ at $72^{\circ} \mathrm{C}$.

PCR product analysis After electrophoresis on denaturing urea gel on an ALF (Automated Laser Fluorescent) sequencer (Amersham-Pharmacia Biotech, Uppsala, Sweden), amplified fragments were directly detected and quantified by the Alfwin Fragment Analyser software package. A modification of the allele ratio in paired tumour and leukocyte DNA is described as $\mathrm{AI}$ (Figure 1). The presence of additional peaks is described as MSI. This technique is highly reproducible and sensitive. As determined previously in our laboratory, a cutoff of $20 \%$ was used to identify significant AI (Schneider et al, 2000). Each alteration was confirmed at least twice by PCR.

\section{Statistics}

All data were computed using SPSS 9.0 for windows (SPSS, Inc. Chicago, IL, USA). The survival function was estimated using Kaplan-Meier test. The log rank to compare survival and the $\chi^{2}$ test were used to analyse correlation between the AI and subgroups of patients.

\section{RESULTS}

We obtained paired blood and tumour biopsies at diagnosis from 54 children. In addition for 13 out of 54 cases, we also collected surgical resection fragments, allowing a comparison between preand postchemotherapy. Characteristics and survival of the entire group are detailed in Table 1. Overall, the event-free survival (EFS) rate at 5 years was $80 \%$. Histological diagnosis showed, in all cases, high-grade osteosarcomas. In all, $30 \mathrm{GR}$ and $23 \mathrm{PR}$ were identified. All the surgical resections were histologically reviewed and all PR patients had less than $95 \%$ tumour necrosis. The statistical analysis of these two groups revealed a significant difference of EFS at 5 years (92 and $69 \%$, respectively, $P=0.01$ ) (Figure 2), associating PR with a worse prognosis. Microsatellite analysis was realised on all samples using six couples of primers. Primer sets were 
Table I Clinical and molecular data of the 54 patients included in our study that are in CCRI or CCR2, in relapse, in partial remission (PR) or dead

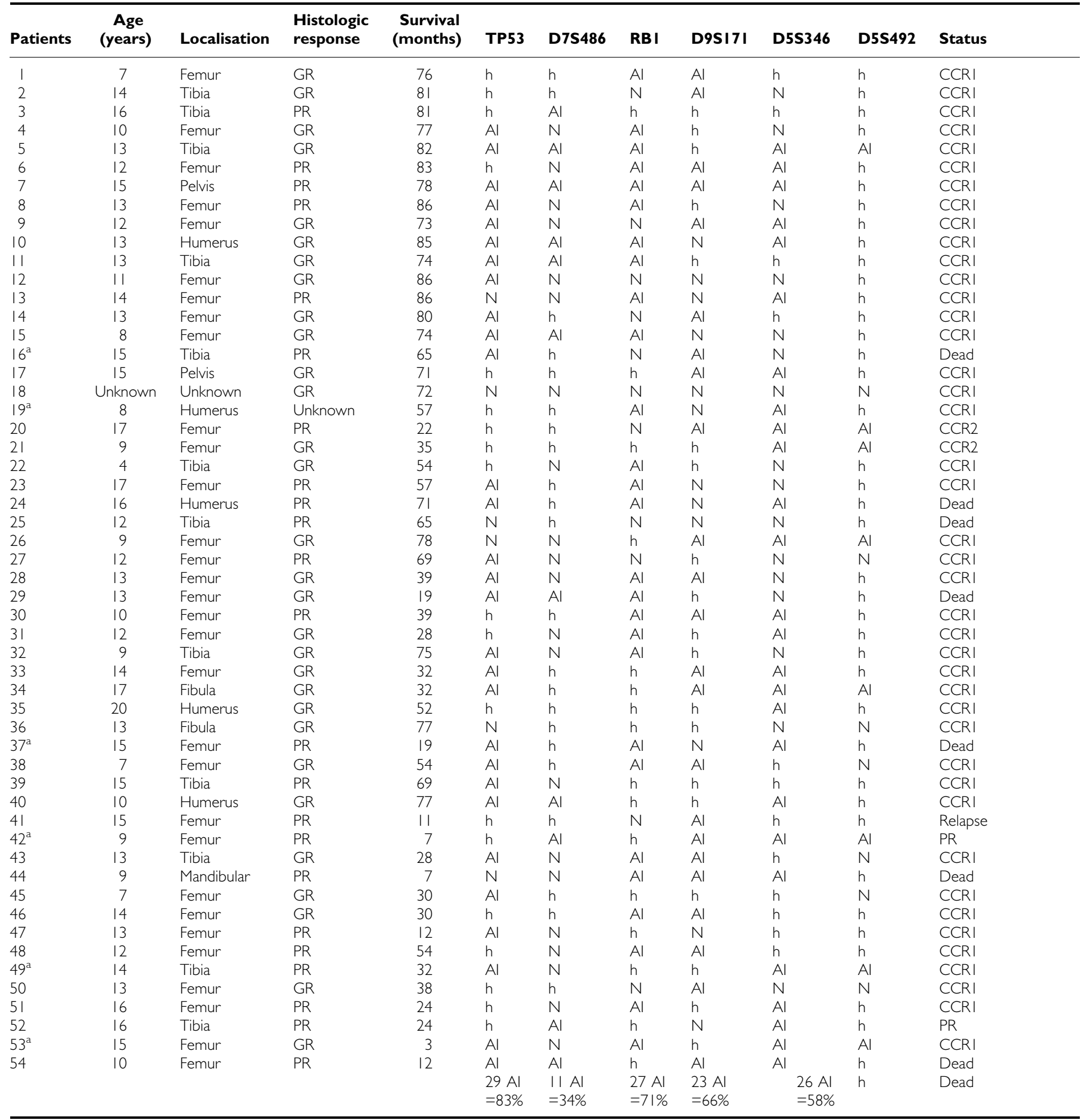

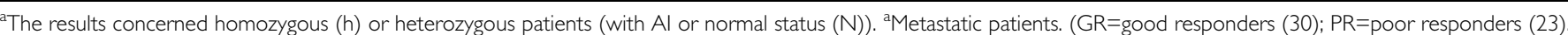

informative for the $\mathrm{p} 53$ gene, localised in $\mathrm{Rb}$ or in $9 \mathrm{p} 21,5 \mathrm{q} 21$ and $7 \mathrm{q} 31$ regions. The last three loci contain, in addition to genes of unknown functions in oncogenesis, genes involved in cell cycle and differentiation, like p16, APC and c-met.

\section{Frequent $\mathrm{AI}$ are observed in biopsies as compared to blood DNA}

The allelotyping did not detect additional peaks (MSI) in our population for our set of six microsatellites. Furthermore, no MSIs were detected at 10 additional microsatellites analysed (D3S700, D3S1283, D4S3019, D4S428, D7S2455, D7S2595, D17S800, D17S1818, D20S107 and D20S855). Thus, it appears that repair error mechanisms were probably not involved in these tumours. In heterozygous patients, a change in the ratio between the two amplified alleles was frequently observed when blood and paired tumour biopsies were compared, indicating the presence of AI. In all, $94 \%$ (51 out of 54) of the patients showed at least one AI, demonstrating that our selection of six microsatellites was highly informative for identifying rearranged primary osteosarcomas at 


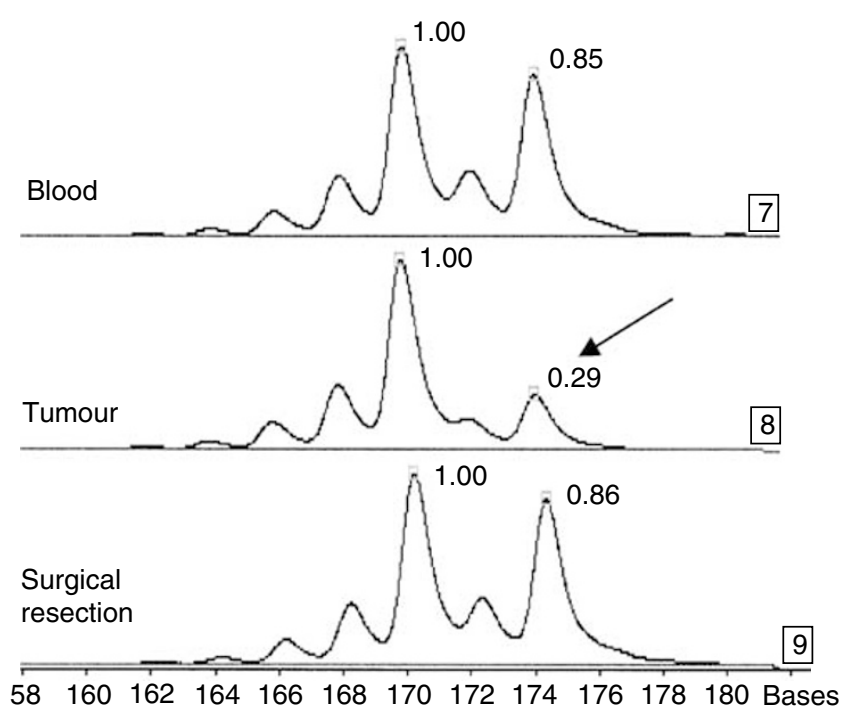

Figure I Allelic imbalance (Al). Genomic DNA was extracted from blood, tumour biopsy at diagnosis and tumour of surgical resection (patient I). PCR of D9SI7I was separated by using a sequencing analyser (ALF Pharmacia). The microsatellite was informative and shows a change in the ratio between the two amplified alleles comparing blood and paired tumour biopsies (black arrow). This patient normalised his surgical tumour sample, there is no change in the ratio between the two amplified alleles comparing blood and paired surgical tumour.

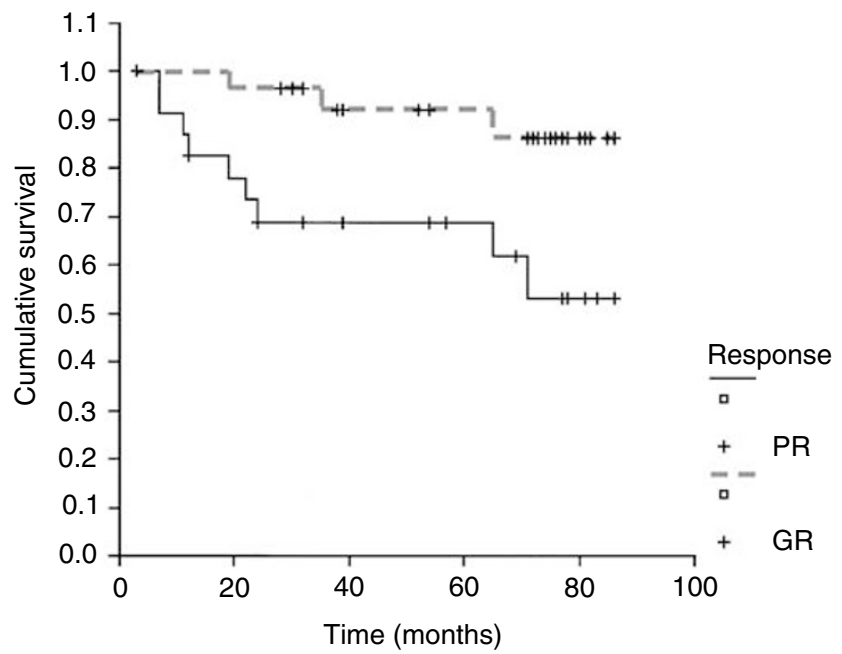

Figure 2 Kaplan-Meier EFS curves for the GR (----) and PR (-) with a statistical significance $(P=0.0 \mathrm{I})$.

diagnosis (Table 1). Samples (three out of 54), lacking microsatellite alterations (patients 18, 25 and 36), showed, in fact, AI at two other microsatellites targeting 3p (D3S1293 and D3S3700), thus confirming the presence of tumour cells. In the informative tumours, we observed a variation of the intensity of the allelic ratio ranging from 35 to $90 \%$ in comparison to normal DNA. This was consistently higher than the previously defined cutoff. These percentages confirmed the high proportion of tumour cells in our biopsies. In each biopsy, the observed allele ratio was at the same intensity for every loci analysed.

A high level of AI frequency was observed at each locus, except for the microsatellite targeting $7 \mathrm{q} 31$. Taking into account heterozygous loci, AI was found in $83 \%$ (29 out of 35 ) for TP53, $71 \%$ (27 out of 38 ) for RB1, 66\% (23 out of 35) for D9S171, 34\% (11 out of 32 ) for D7S486 and 58\% (26 out of 45) for D5S346 and

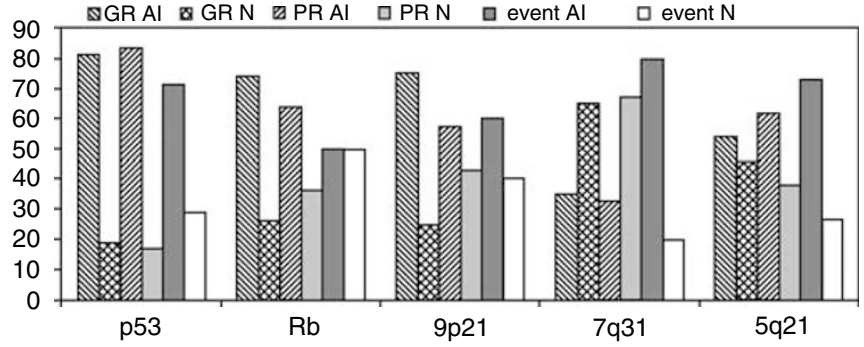

Figure 3 Allelic imbalance (Al) frequencies and percentages of normal (N) status at the five targeted loci in the different subgroups of patients: GR, PR and the population combining different events such as partia remission, relapse and deaths (event).

D5S492 (Table 1). Furthermore, a slightly higher proportion of homozygous loci, than reported in the database, was observed at D7S486 (41 vs 35\% for TP53, 30\% for RB1, 35\% for D $9 S 171$ and $17 \%$ for $5 \mathrm{q} 21)$. At least one of the cell cycle genes, p53, Rb or locus 9 p21, was altered in $88 \%$ biopsies. In order to analyse the data at the locus containing APC, we used two microsatellites flanking this region (D5S346 and D5S492), and considered this locus as informative if it was heterozygous for both markers or if at least one of the two markers was modified, and the second one noninformative. In cases where both microsatellites (D5S346 and D55492) were informative, results at both sites were always identical, either both AI or both normal. In GR and PR subgroups, AI frequencies for each microsatellite did not show statistical differences (Figure 3). In the subgroup, combining relapses, partial responses to treatment or dead patients (patients 16, 24, 25, 29, 37, $41,42,44,52$ and 54), a higher AI frequency was observed at D7S486 and at D5S3546 and D5S492, but a statistical significance was found only for the 7q31 locus $(P=0.04)$ (Figure 3$)$.

Event-free survival was investigated for each microsatellite in the entire population. No significant differences in survival time were observed to be related to the presence of AI at TP53, RB1 or D9S171. Interestingly, a normal status at $7 \mathrm{q} 31$ was associated with a significantly better EFS not only in the whole population, but also in the PR subgroup (Figure 4$)(P=0.03)$. The association of a rearrangement at markers flanking $5 \mathrm{q} 21$ with a bad prognosis is suggested by the curves, but the correlation is not statistically significant $(P=0.15)$ (Figure 5).

\section{Comparison of tumour samples at diagnosis with the surgical resection following chemotherapy}

Fortunately, we were able to collect a surgical sample in addition to biopsy for 13 cases (nine PR and five GR) (Table 2). In three out of five GR, the presence of AI was still observed (patients 4, 10 and 29). All five GR patients had between 95 and $99 \%$ necrosis. However, the allotyping analysis allowed us to identify four different groups.

First, four children (patients 1, 3, 11, 49), comprising two GR and two PR, normalised their surgical resection, probably suggesting the absence of any significant tumour cell population according to our molecular detection. These four patients are alive and in CCR1. In the second group (one PR and one GR, cases 6 and 10 ), the alterations at $7 \mathrm{q} 31$ and $5 \mathrm{q} 21$ regions persisted, but the other AI previously identified were no longer observed. The third group concerned four patients (cases $4,8,27,54$ ), who showed the same rearrangements in both biopsy and tumour resection. For these four patients, our molecular analyses suggested an initial significant chemoresistance of the localised tumour cell population, even though one patient was classified as GR in CCR1. Finally, in the last group (patients 16, 29, 47), new microsatellite alterations appeared in comparison with biopsy, which were localised at the locus $5 \mathrm{q} 21$ for patient 16 (PR) and 29 (GR). Both 
A

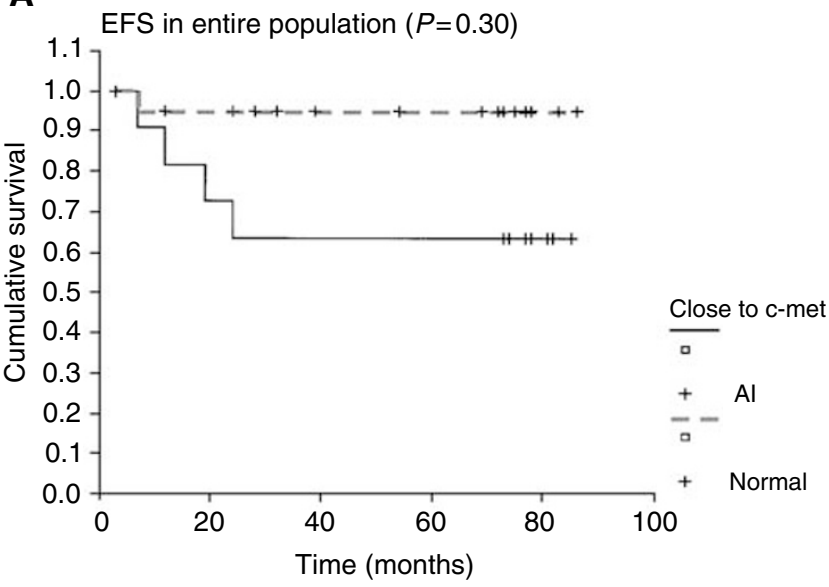

B

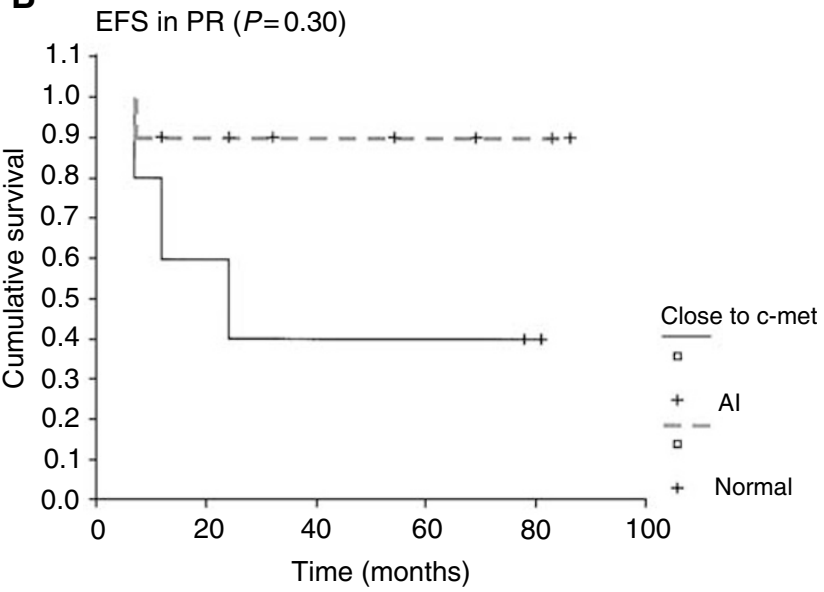

Figure 4 Kaplan-Meier EFS curves of the microsatellite analysis at 7q31 locus for the entire population $(\mathbf{A})$ and for the PR (B) with a statistically significant association between the presence of $\mathrm{Al}$ and a worse prognosis $(-)$.

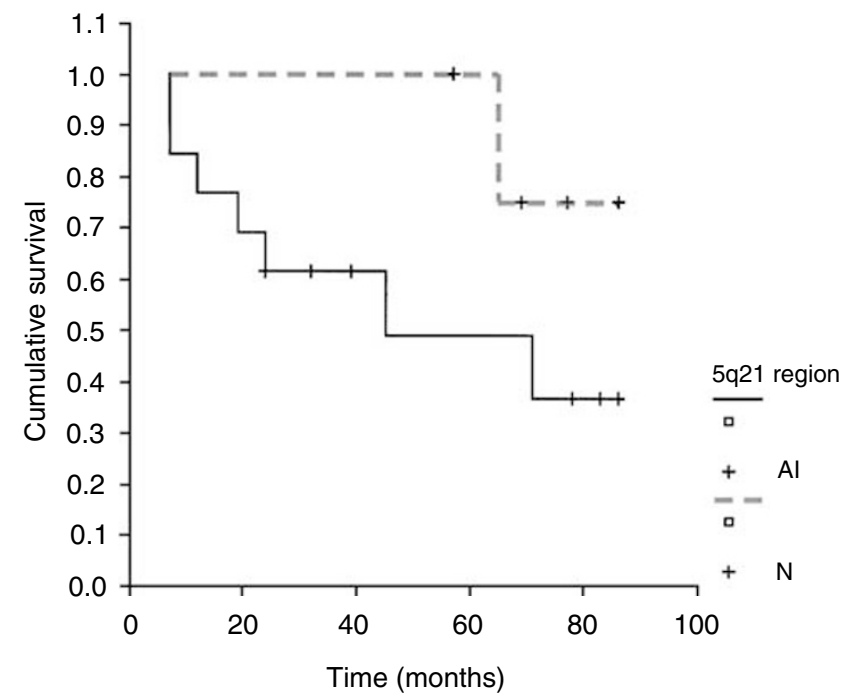

Figure 5 Kaplan-Meier EFS curve for the PR with $\mathrm{Al}$ at $5 \mathrm{q} 2 \mathrm{I}$ region and without alterations. This statistical analysis showed a trend between the presence of $\mathrm{Al}$ and a worse survival.

patients died. Histological response was not correlated to the results of microsatellite analysis in any of the groups. In the relapsing and metastatic specimens, we detected the same alterations, as observed in biopsy or surgical samples, suggesting the evolution of the same tumour clone (Table 2).

\section{DISCUSSION}

\section{Overall considerations about our biopsy collection}

This study was designed to identify molecular prognostic factors assessable at diagnosis, as $\mathrm{Rb}$ and $\mathrm{p} 53$ genes, $9 \mathrm{p} 21$ and $7 \mathrm{q} 31$ loci and a region surrounding the APC gene. Candidate genes in $9 \mathrm{p} 21$ and $7 \mathrm{q} 31$ are p16 and c-met, respectively. Altogether, clinical and statistical data of our population appeared to be representative of the usual histological diagnoses and treatment responses in the multicentre OS 94 protocol. The main difficulty was obtaining paired blood and tumour DNA samples, mainly because of the low incidence of such sarcomas. Despite this problem, our study included 54 primary paediatric high-grade osteosarcomas, treated with the same therapeutic protocol. Previous published studies concerned either adult or paediatric populations, mixed the lowor high-grade osteosarcomas and had different therapeutic regimens (Ozisil et al, 1994; Patino-Garcia and Sierrasesumaga, 1997; Nielsen et al, 1998; Tsuchiya et al, 2000). To our knowledge, no other molecular study has investigated such a large homogeneous paediatric population.

Our microsatellite analysis was achieved in 54 paired blood and fresh-frozen biopsy specimens. Allelic imbalances were detected, but there were no MSI, allowing us to infer that the RER phenotype (Fearon and Vogelstein, 1990; Kinzler and Vogelstein, 1996; Hanahan and Weinberg, 2000) is not the main mechanism involved in the oncogenesis of osteosarcoma. Belchis et al (1996) showed MSI at the retinoblastoma locus in eight out of 18 informative osteosarcomas, but their study investigated formalinfixed, paraffin-embedded samples, which could affect microsatellite analyses. With this panel of six microsatellites, $94 \%$ of our population showed DNA alterations at diagnosis. This high frequency recommends the choice of this sensitive and powerful method and these selected markers for routine analyses at diagnosis. Furthermore, our results showed a high intensity of allelic ratio alterations, confirming the high percentages of tumour cells in these biopsies. The clonal homogeneity of tumour samples was confirmed by the same high percentage allelic ratio intensity at each locus analysed from the same tumour.

\section{Analysis of results and comparison of biopsy DNA samples and paired blood DNA for the loci involved in the cell cycle}

With this sensitive technique, high $\mathrm{AI}$ frequencies in $\mathrm{p} 53, \mathrm{Rb}$ and 9 p21 loci were observed. These high frequencies of alterations at TP53 and 9p21 (locus containing CDK inhibitor INKAp16) were in agreement with previous data (Nielsen et al, 1998; Tsuchiya et al, 2000; Benassi et al, 2001). Several studies concerning these genes have reported lower frequencies, however they used either protein level analysis or mutation detection and are therefore difficult to compare with microsatellite analysis. In contrast with these studies, we did not find any correlation between the presence of $\mathrm{AI}$ and prognosis (Nielsen et al, 1998; Maitra et al, 2001).

Concerning the Rb locus, the same frequency (70\%) of AI was found as in the previous SFOP study (Feugeas et al, 1996). According to the previous report, there was no significant correlation between the abnormalities at this locus and the histological grading in our present study (Feugeas et al, 1996). In contrast, in our study no correlation between EFS and AI at RB1 was observed, while the previous study suggested correlation of $\mathrm{Rb}$ locus alteration and poor prognosis. Probably, such discrepancy could be explained by the modifications of the chemotherapy between these two protocols. In fact, we analysed a different prognostic group of patients, since in our study 30 GR and 23 PR 
Table 2 Molecular analysis of the 13 patients whose normal tissue, biopsy at diagnosis and surgical resection samples were analysed

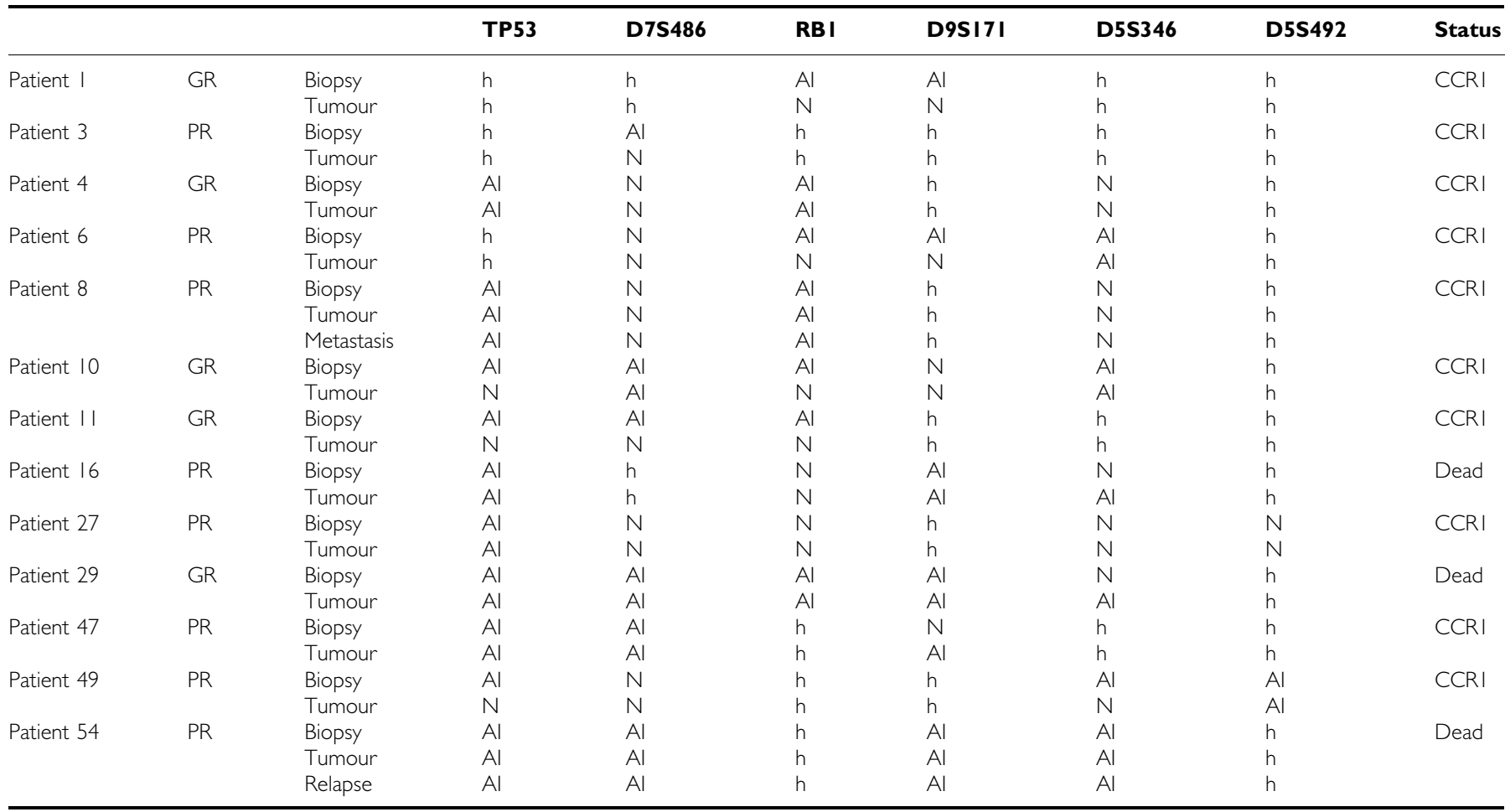

The results were homozygous status (h), Al or normal status (N). This study involved GR and PR that were in first CCRI or dead.

were explored $v s 12 \mathrm{GR}$ and $19 \mathrm{PR}$ in the previous one (Feugeas et al, 1996).

Altogether, our results, like previous data, confirmed the putative role of $9 \mathrm{p} 21$ region and $\mathrm{Rb} / \mathrm{p} 53$ signalling pathway in osteosarcomas (Nielsen et al, 1998; Tsuchiya et al, 2000; Benassi et al, 2001). However, the lack of correlation between survival and the presence of alteration at those loci suggested that other mechanisms are involved in the sensitivity to chemotherapy.

\section{Microsatellite analysis of 5q21 and 7q31: a prognostic marker and a new probable target diagnosis}

Results at the locus $7 \mathrm{q} 31$ and at the region containing APC suggested a striking and interesting role for these sites in osteosarcoma oncogenesis, even though the frequencies of rearrangements at the locus $7 q 31$ were rather low (34\%) (Table 1). Nevertheless, when we only considered the group comprising partial remissions, relapses and patients who died, this AI frequency reached $80 \%$, resulting in a significant correlation with this bad prognostic subgroup $(P=0.04)$. Thus, our data have identified a new region involved in the prognosis of osteosarcomas. The significant correlation between alteration of $7 q 31$ and a worse prognosis demonstrates its prognostic role. Results obtained at this locus were in agreement with previous c-met studies, concluding that c-met could play a role in aggressive osteosarcomas (Ferracini et al, 1995; Oda et al, 2000). Even though such results should be confirmed in a larger population with a longer follow-up and a c-met gene quantitative analysis, the knowledge of the presence of AI at this locus could be useful in predicting GRs that will relapse and might encourage the use of kinase inhibitors in osteosarcomas treatments in future.

At the locus 5q21, the use of two microsatellites, surrounding the APC gene, increased the specificity of allelotyping at this site and allowed us to speculate that the high percentage of rearrangement could strengthen the fundamental role of this gene in osteosarcoma. To our knowledge, such results in osteosarcomas have only been described in one recent study (Monaghan et al, 2001), since APC has previously been known to play a role in the oncogenesis of colorectal cancer (Polakis, 1997). Furthermore, statistical analyses showed a slight trend for the presence of AI at $5 \mathrm{q} 21$ correlating with the poor response to chemotherapy, in agreement with a trend towards a worse prognosis in case of AI at 5 q21. However, a larger cohort would be required to confirm this. In addition, further studies would be necessary to define precisely the status of this gene or the presence of mutation.

\section{Allelotyping surgical resection specimens as additional tool for refining the histological response to preoperative chemotherapy}

Since some GR are known to relapse earlier, refining the stratification of GR could help to adjust treatment. Our allelotyping of surgical resections was very informative, allowing us to characterise four subgroups that did not appear to be correlated with histological response. In the groups where AI were still detected in the surgical specimens, we identified two situation: either same alterations or new AI were observed in surgical samples compared to biopsies. The second situation suggested the presence of new cellular clones, possibly selected by chemotherapy since the reproducibility and high intensity of AI agreed strongly with the presence of a dominant cell clone at diagnosis. It should be noted that the patients who died (patients 16 and 29) had additional $\mathrm{AI}$ at $5 \mathrm{q} 21$, strengthening the role of this loci in poor prognosis contrary to the case in colorectal cancer (Polakis, 1997). In fact, only GR with persisting AI should be carefully followed since these GR could belong to the subgroup who could relapse earlier. Altogether, allelotyping at surgical resection time could complete the histological analysis and help to provide a more precise stratification of each subgroup of responders.

In conclusion, our large population of 54 paediatric osteosarcomas was investigated with five targeted loci. These five loci 
proved to be good diagnostic markers in at least $94 \%$ of samples and revealed a potential implication of $5 \mathrm{q} 21$ region containing APC. A significant correlation between presence of rearrangements at $7 \mathrm{q} 31$ and a worse prognosis was observed. Finally, the use of targeted microsatellite analysis at surgical resection could be an additional tool to make more precise stratification, especially in GR.

\section{REFERENCES}

Alonso J, Garcia-Miguel P, Abelairas J, Mendiola M, Pestana A (2001) A microsatellite fluorescent method for linkage analysis in familial retinoblastoma and deletion detection at the RB1 locus in retinoblastoma and osteosarcoma. Diagn Mol Pathol 10: 9-14

Belchis DA, Meece CA, Benko FA, Rogan PK, Williams RA, Gocke CD (1996) Loss of heterozygosity and microsatellite instability at the retinoblastoma locus in osteosarcomas. Diagn Mol Pathol 5: 214-219

Benassi MS, Molendini L, Gamberi G, Magagnoli G, Ragazzini P, Gobbi GA, Sangiorgi L, Pazzaglia L, Asp J, Bransting C, Picci P (2001) Involvement of INK4A gene products in the pathogenesis and development of human osteosarcoma. Cancer 92: $3062-3067$

Dahlin DC, Unni KK (1986) In Bone Tumors, General Aspects and Data on 8542 Cases, Charles CT (ed) pp 227-307. Ilinois: Springfield, IL

Duffaud F, Digue L, Baciuchka-Palmaro M, Volot F, Perles-Daniel C, Garbe L, Favre R (2000) Osteosarcomas of flat bones in adolescents and adults. Cancer 88: $324-332$

Fearon ER, Vogelstein B (1990) A genetic model for colorectal tumorigenesis. Cell 61: 759-767

Ferracini R, Di Renzo MF, Scotlandi K, Baldini N, Olivero M, Lollini P, Cremona O, Campanacci M, Comoglio PM (1995) The met/HGF receptor is over-expressed in human osteosarcomas and is activated by either a paracrine or an autocrine circuit. Oncogene 10: 739-749

Feugeas O, Guriec N, Babin-Boilletot A, Marcellin L, Simon P, Babin S, Thyss A, Hofman P, Terrier P, Kalifa C, Brunat-Mentigny M, Patricot LM, Oberling F (1996) Loss of heterozygosity of the $\mathrm{Rb}$ gene is a poor prognostic factor in patients with osteosarcoma. J Clin Oncol 14: 467472

Gadwal SR, Gannon FH, Fanburg-Smith JC, Becoskie EM, Thompson LDR (2001) Primary osteosarcoma of the head and neck in pediatric patients. Cancer 91: 598-605

Hanahan D, Weinberg RA (2000) The hallmarks of cancer. Cell 100: $57-70$

Huvos AG (1991) Bone Tumors, Diagnosis, Treatment, and Prognosis, 2nd edn Philadelphia: W.B. Saunders,, pp 85-155, 343-393

Huvos AG, Rosen G, Marcove RC (1977) Primary osteogenic sarcoma. Pathologic aspects in twenty patients after treatment with chemotherapy, in bloc resection and prosthetic bone replacement. Arch Pathol Lab Med 101: $14-18$

Karran P (1996) Microsatellite instability and DNA mismatch repair in human cancer. Cancer Biol 7: 15-24

Kinzler KW, Vogelstein B (1996) Lessons from hereditary colorectal cancer. Cell 87: $159-170$

Maitra A, Roberts H, Weinberg AG, Geradts J (2001) Loss of p16 expression correlates with decreased survival in pediatric osteosarcomas. Int $J$ Cancer 95: 34-38
Malkin D, Li FP, Strong LC, Fraumeni JFJ, Nelson CE, Kim DH, Kassel J, Gryka MA, Bischoff FZ, Tainsky MA (1990) Germline p53 mutations in a familial syndrome of breast cancer, sarcomas, and other neoplams. Science 250: $1233-1238$

Mertens F, Mandahl N, Baldetorp B, Bauer HCF, Ryholm A, Wiebe T (1993) Cytogenetic findings in 33 osteosarcomas. Int $J$ cancer 55: 44-50

Miller SA, Dykes DD, Polesky HF (1988) A simple salting out procedure for extracting DNA from human nucleated cells. Nucleic Acid Res 16: 1215

Monaghan H, Bubb VJ, Sirimujalin R, Millward-Sadler SJ, Salter DM (2001) Adenomatous polyposis coli (APC), beta-catenin, and cadherin are expressed in human bone and cartilage. Histopathology 39: 611-619

Nagler RM, Malkin L, Ben-Arich Y, Laufer D (2000) Sarcoma of the maxillofacial region: follow-up of 25 cases. Anticancer Res 20: $3735-3745$

Nielsen GP, Burns KL, Rosenberg AE, Louis DN (1998) CDKN2A gene deletions and loss of p16 expression occur in osteosarcomas that lack RB alterations. Am J Pathol 153: 159-163

Oda Y, Naka T, Takesita M, Iwamoto Y, Tsuneyoshi M (2000) Comparison of histological changes and changes in $\mathrm{nm} 23$ and c-met expression between primary and metastatic sites in osteosarcoma: a clinicopathologic and immunohistochemical study. Hum Pathol 31: 709-716

Ozisil YY, Meloni AM, Peier A, Altungoz O, Spannier AS, Zalupski MM, Leong SP, Sandberg AA (1994) Cytogenetic findings in 19 malignant bone tumors. Cancer 74: 2268-2275

Park YB, Kim HS, Oh JH, Lee SH (2001) The co-expression of p53 protein and P-glycoprotein is correlated to a poor prognosis in osteosarcoma. Int Orthop 24: $307-310$

Patino-Garcia A, Sierrasesumaga L (1997) Analysis of the p16INK4 and TP53 tumor suppressor genes in bone sarcoma pediatric patients. Cancer Genet Cytogenet 98: 50-55

Polakis P (1997) The adenomatous polyposis coli (APC) tumor suppressor. Biochim Biophys Acta 1332: $127-147$

Schneider A, Borgnat S, Lang H, Regine O, Linfner V, Kassem M, Saussine C, Oudet P, Jacqmin D, Gaub MP (2000) Evaluation of microsatellite analysis in urine sediment for diagnosis of bladder cancer. Cancer Res 60: 4617-4622

Tsuchiya T, Sekine K, Hinohara S, namiki T, Nobori T, Kaneko Y (2000) Analysis of the p16INK4, p14ARF, p15, TP53, and MDM2 genes and their prognostic implications in osteosarcoma and Ewing sarcoma. Cancer Genet Cytogenet 120: $91-98$

Wadayama BI, Toguchida J, Shimizu T, Ishizaki K, Sasaki MS, Kotoura Y, Yamamuro T (1994) Mutation spectrum of the retinoblastoma gene in osteosarcomas. Cancer Res 54: $3042-3048$ 